\title{
Compact Slanted Grating Couplers Between Optical Fiber and InP-InGaAsP Waveguides
}

\author{
Frederik Van Laere, Student Member, IEEE, Maria V. Kotlyar, Dirk Taillaert, Member, IEEE, \\ Dries Van Thourhout, Member, IEEE, Thomas F. Krauss, and Roel Baets, Fellow, IEEE
}

\begin{abstract}
We present theoretical and experimental results for compact slanted gratings for vertical coupling between single-mode fiber and InP-InGaAsP waveguides. The maximum calculated coupling efficiency is $59 \%$. We have measured a coupling efficiency of $16 \%$ for a $10-\mu \mathrm{m}$-long slanted grating.
\end{abstract}

Index Terms-Chemically assisted ion beam etching, InP-InGaAsP waveguides, integrated optics, slanted grating.

\section{INTRODUCTION}

$\mathbf{E}$ FFICIENT coupling between fiber and thin-film waveguides on chip is an important problem in optical communication networks. The mismatch between fiber mode and waveguide mode results in large insertion losses and high packaging cost.

Established solutions use edge-coupling and are often taper-based [1], [2]. Another approach uses grating couplers for out-of-plane coupling between fiber and waveguides on chip. Light can be coupled in and out everywhere on the chip, opening the way to wafer-scale testing. Compact and efficient rectangular grating couplers in silicon-on-insulator (SOI) have been demonstrated in [3]. However, SOI is not very well suited for making active components, where InP is preferred.

However, the vertical index contrast of InP-based layer structures is typically too low for designing these strong and compact rectangular gratings. One solution is using high vertical index contrast InP membranes for the couplers [4] and other passive components, which then have to be integrated with active components in classical InP-based layer structures.

Another possibility is to use slanted gratings instead of rectangular ones. Very high theoretical coupling efficiencies were reported for compact slanted grating couplers, both for low vertical index contrast polymeric waveguides (80.1\%) [5] and high

Manuscript received October 16, 2006; revised December 29, 2006. This work was supported in part by the European Union under the IST Project FUNFOX. Part of this work was performed in the framework of the Belgian IAP Photon Network. The work of F. Van Laere was supported by the Institute for the Promotion of Innovation through Science and Technology (IWT Flanders) under a scholarship. The work of D. Taillaert was supported by the Institute for the Promotion of Innovation through Science and Technology (IWT Flanders) under a postdoctoral grant.

F. Van Laere, D. Taillaert, D. Van Thouhout, and R. Baets are with the Department of Information Technology (INTEC), Ghent University-IMEC, B-9000 Gent, Belgium (e-mail: frederik.vanlaere@intec.ugent.be).

M. V. Kotlyar and T. F. Krauss are with the School of Physics and Astronomy, University of St. Andews, St. Andrews, Fife KY16 9SS, U.K.

Color versions of one or more of the figures in this letter are available online at http://ieeexplore.ieee.org.

Digital Object Identifier 10.1109/LPT.2007.891975

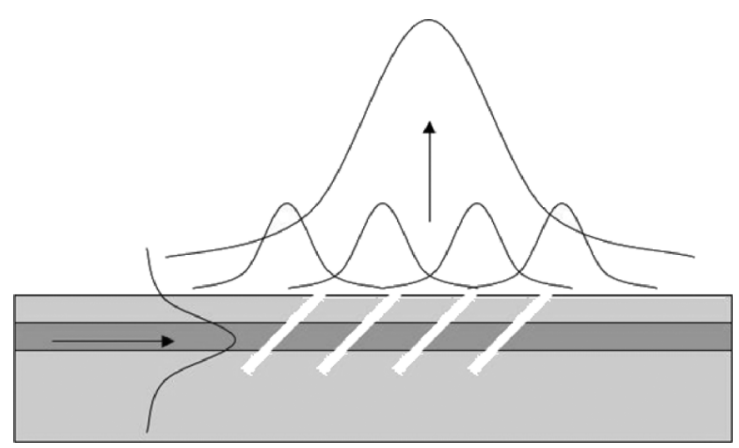

Fig. 1. Principle of the slanted grating coupler.

vertical index contrast SOI waveguides (75.8\%) [6], but no experimental results have been reported as yet. It would be of great interest if this type of slanted couplers can be implemented in an active layer structure. Surface-emitting lasers in InGaAs-AlGaAs with blazed grating outcouplers have been demonstrated in [7]. The surface emission efficiency of the lasers, using long $(320 \mu \mathrm{m})$ grating outcouplers, was measured to be over $80 \%$.

We have designed and fabricated very compact $(10 \mu \mathrm{m})$ slanted grating couplers in a InP-InGaAsP heterostructure for coupling between standard single-mode fiber and waveguides. We report an experimental coupling efficiency to fiber of $16 \%$ for nonoptimized structures.

\section{PRINCIPLE}

In this section, we describe the device as an output coupler (Fig. 1). The operation as an input coupler is analogous.

By etching air slots at an angle of $45^{\circ}$, the vertical (outcoupling) direction is favored. Etching through the core is required to capture all of the mode. The incident light reflects upwards at the interface with air. If the slots are narrow enough, part of the light can tunnel through the slot and reach a second slot, where again it gets partly reflected and partly transmitted. In this way, the waveguide mode is coupled out vertically in a distributed way, in order to match the mode of a fiber positioned vertically above the grating. By varying the coupling strength of each slot, a desired mode profile can be created, e.g., to match the Gaussian profile of a fiber mode, although this has not been implemented yet.

\section{DESIGN}

\section{A. Method}

The design of the gratings is carried out in CAMFR, an eigenmode expansion tool [8]. The slanted walls are approximated 


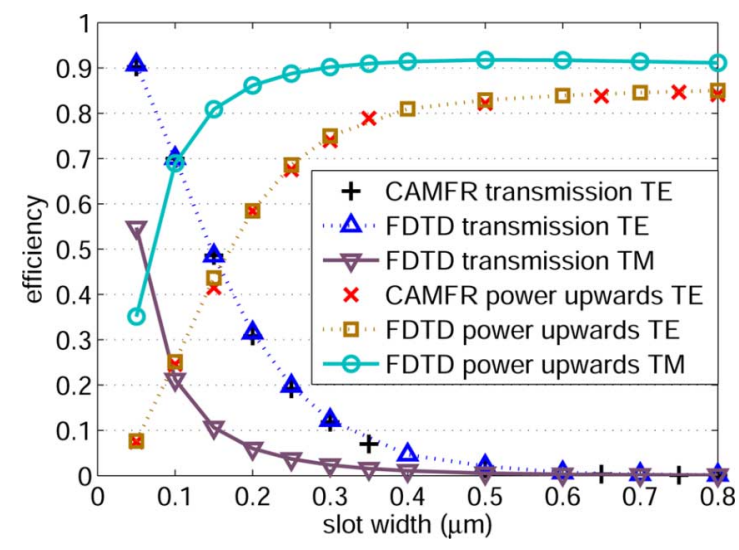

Fig. 2. Radiation properties of one single slot, etched at an angle of $45^{\circ} \mathrm{com}-$ pletely through the waveguide core.

by discretizing them in narrow slabs, for which the eigenmodes have to be calculated. We consider the coupling from waveguide to fiber, i.e., coupling from one mode to another. The 3 -D problem can be approximated by a 2-D problem, since the lateral dimensions are much larger than the wavelength. The coupling efficiency is calculated using an overlap integral between the outcoupled field profile and a Gaussian fiber mode profile (diameter $10.4 \mu \mathrm{m}$ ). The coupling efficiency of the 2-D problem is multiplied by a correction factor to take into account the lateral direction. The design method of grating couplers with eigenmode expansion is described in detail in [3]. We have verified all simulation results with 2-D finite-difference time-domain (FDTD) simulations, using a grid of $10 \mathrm{~nm}$.

\section{B. Radiation Properties of a Single Slot}

The most important parameter in the design is the width of the air slots. When the slots are too wide, there will be little transmission (tunneling), and almost all the light will be coupled out by the first slot, resulting in a poor overlap with the fiber mode. When the slots are too narrow, most of the light will tunnel through the slot, and little light will be reflected upwards. Two-dimensional simulation results for a single slot, etched at $45^{\circ}$ completely through the core, are shown in Fig. 2. The transmitted and upwards radiated power is calculated as a function of slot width. The waveguide has a 522-nm-thick InGaAsP (Q1.22) core and a 300-nm InP-top cladding, on an InP substrate. A top antireflection (AR) coating (a quarter-wave layer) with $n=\operatorname{sqrt}\left(n_{\mathrm{InP}}\right)$ is applied in order to avoid radiation back into the waveguide. The wavelength is $1550 \mathrm{~nm}$ and the polarization is transverse electric [(TE) electric field parallel to the slots]. A very good agreement is obtained with 2-D FDTD simulations. The upwards radiated power and transmitted power do not add up to $100 \%$, because part of the power is scattered and radiated towards the substrate.

We have also calculated the radiation properties for transverse-magnetic (TM) polarization (magnetic field parallel to the slots). For narrow slots, substantially more light is radiated upwards than in the TE case, so it is difficult to couple light out in a distributed way for achieving high coupling efficiency. These results indicate that the slanted coupler is not polarization-insensitive. From now on, we therefore only consider TE polarization.

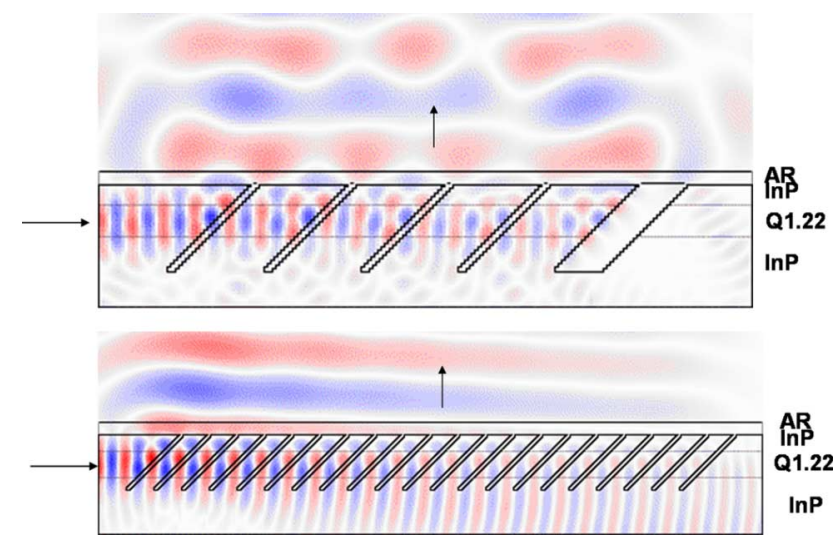

Fig. 3. Field profile of slanted couplers calculated with CAMFR. (top) Using third diffraction order. (bottom) Using first diffraction order.

\section{Slanted Grating Couplers}

In a first design, the third diffraction order is coupled into the fiber. We use four slots of width $150 \mathrm{~nm}$, etched to a depth of $1.3 \mu \mathrm{m}$ (vertically). To couple all the remaining light upwards, the last slot is $700 \mathrm{~nm}$ wide. The period is $1.51 \mu \mathrm{m}$ and has been optimized to obtain a phase front propagating in the vertical direction. The field distribution calculated for this structure is shown in Fig. 3 (top). The maximum coupling efficiency to a single-mode fiber of the 2-D simulation problem is $34 \%$ without AR coating and $48 \%$ with AR coating. The coupling efficiency can be further increased by individually optimizing each slot and the spacing in between them (e.g., by genetic algorithms) [3], [5]. In a second design, we operate on the first diffraction order, which makes the period smaller and requires more periods to match the dimensions of the fiber core. This implies that the slots should be narrower, which is difficult to fabricate, so we choose 50-nm slots as a lower bound. The etch depth is $1 \mu \mathrm{m}$ (vertically), a realistic value from a fabrication point of view. However, deeper etching is preferred to avoid coupling towards the substrate. The final structure has 20 periods (period is $0.49 \mu \mathrm{m}$ ), resulting in a maximum coupling efficiency to fiber (2-D problem) of $43 \%$ and $59 \%$ with and without AR coating, respectively. The field distribution is shown in Fig. 3 (bottom). The calculated 1-dB bandwidth of these grating couplers is around $60 \mathrm{~nm}$.

\section{FABRICATION}

The layer structure consists of an InP substrate, a 522-nm InGaAsP layer $\left(\lambda_{g}=1.22 \mu \mathrm{m}\right)$, and a 300-nm InP top-cladding. The gratings are defined by e-beam lithography and transferred into a $\mathrm{SiO}_{2}$ hard mask by reactive ion etching using $\mathrm{CHF}_{3}$. Finally, the structures are etched into the epistructure by means of chemically assisted ion beam etching using $\mathrm{Cl}_{2}$ [9]. The sample is mounted on a slanted holder in order to etch the slots at the desired angle of $45^{\circ}$. Afterwards, $4-\mu \mathrm{m}$-wide waveguides are defined using optical lithography, and etched to a depth of $70 \mathrm{~nm}$. Because of this shallow etch depth, the mode is only weakly confined, and has a good lateral overlap with the fiber mode.

\section{Measurements}

In order to measure the coupling efficiency, an input fiber, connected with a tunable laser, is positioned vertically at a height of approximately $10 \mu \mathrm{m}$ above the grating. The light 


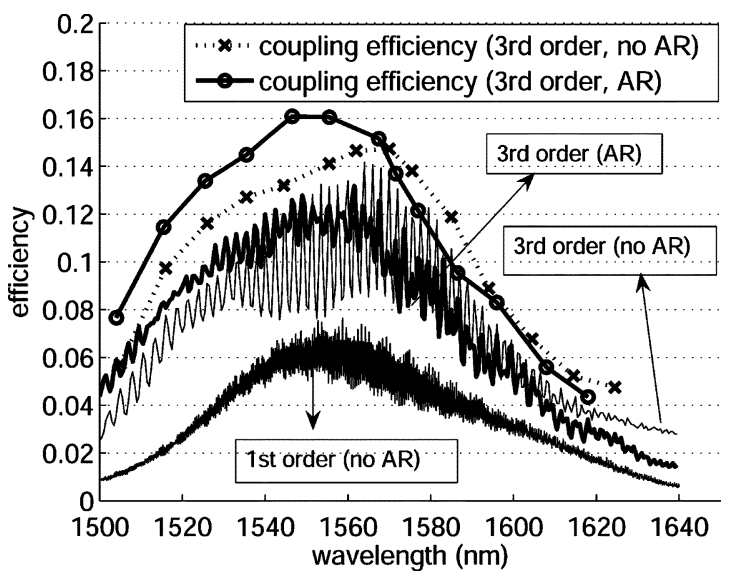

Fig. 4. Measurement results on slanted grating couplers. Transmission efficiency curves and calculated coupling efficiency curves are shown.
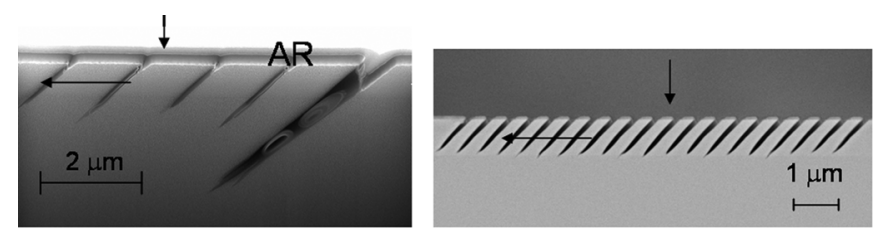

Fig. 5. SEM pictures of fabricated structures. Arrows indicate the coupling direction of the measurements (input coupler). (left) Third-order slanted coupler with $\mathrm{Al}_{2} \mathrm{O}_{3}$ AR coating. (right) First-order slanted coupler.

coupled to a waveguide is collected at the cleaved facet of the chip and measured by a detector. The measurement results are summarized in Fig. 4.

The coupling efficiency is calculated from the ratio between measured power and input power (transmission efficiency). In these calculations, Fabry-Pérot cavity effects between grating and output facet have to be taken into account. First, we calculate the transmission of a cavity with the (fixed and known) facet reflectivity and the (yet unknown) grating reflectivity as a parameter. The grating reflectivity is then determined by matching the modulation depth of the measured Fabry-Pérot fringes with the calculated ones. From the calculated cavity transmission, a correction factor is determined for defining the relation between coupling efficiency and measured transmission efficiency.

First, we have measured the transmission efficiency of the third-order slanted coupler. From this measurement, the coupling efficiency is determined to be $14 \%$ for the case without AR coating (dotted line, $\mathrm{x}$-marker). Afterwards, an $\mathrm{Al}_{2} \mathrm{O}_{3} \mathrm{AR}$ coating is deposited using e-gun evaporation. In this case, the depth of the Fabry-Pérot fringes is smaller, as expected. The coupling efficiency is slightly increased to $16 \%$ (solid line, o-marker).

We have also fabricated the first-order design (only without AR coating). The experimental coupling efficiency, determined from the measured transmission, is $8 \%$. Scanning electron microscope (SEM) pictures of a cross section of both couplers are shown in Fig. 5. Some voids originating from depositing an AR coating $\left(\mathrm{Al}_{2} \mathrm{O}_{3}\right)$ on a nonplanar structure can be seen.

The measurements show a rather large deviation from the optimal calculated coupling efficiency. However, the fabrication of the devices is very challenging. It is very difficult to etch narrow slots $(50$ or $150 \mathrm{~nm})$ very deep $(1.0 \mu \mathrm{m}$ and preferably more) at an angle. This etch depth is required to capture all of the mode and radiate the power upwards. In addition, if the slots are wider than designed, most of the light is coupled out by only the first few slots, resulting in a poor overlap with the fiber mode. For the actual fabricated first-order coupler (vertical etch depth of $850 \mathrm{~nm}$, slot width of $70 \mathrm{~nm}$ ), the calculated coupling efficiency (no AR coating) is $28 \%$. For the actual fabricated third-order coupler (vertical etch depth of $1.1 \mu \mathrm{m}$ and slot width of $138 \mathrm{~nm}$, except for the last one), the calculated coupling efficiency is 33\% (without AR coating) and $45 \%$ (with $267 \mathrm{Al}_{2} \mathrm{O}_{3}(n=1.57)$ as AR coating). Currently, work is in progress to design couplers with relaxed fabrication tolerances, and the fabrication process is being further optimized.

\section{CONCLUSION}

We have designed and fabricated compact $(10 \mu \mathrm{m})$ slanted grating couplers between single-mode fiber and InP-InGaAsP waveguides. We have measured $16 \%$ coupling efficiency on first fabricated, nonoptimized components. These are, to our knowledge, the first experimental results reported for this type of compact coupler. By optimizing the grating parameters (e.g., using genetic algorithms), the coupling efficiency can be increased substantially. We believe that vertical coupling and the straightforward integration of this component with active devices can be of great interest for use in future telecommunication networks.

\section{ACKNOWLEDGMENT}

Alcatel-Thales III-V Laboratory is acknowledged for providing the InP epiwafers. J. Schrauwen is acknowledged for making FIB cross sections on fabricated structures.

\section{REFERENCES}

[1] Y. J. Yan, M. L. Masanovic, E. J. Skogen, Z. Hu, D. J. Blumenthal, and L. A. Coldren, "Optical mode converter integration with InP-InGaAsP active and passive waveguides using a single regrowth process," IEEE Photon. Technol. Lett., vol. 14, no. 9, pp. 1249-1251, Sep. 2002.

[2] T. Shoji, T. Tsuchizawa, T. Watanabe, K. Yamada, and H. Morita, "Low loss mode size converter from $0.3 \mu \mathrm{m}$ square $\mathrm{Si}$ wire waveguides to singlemode fibres," Electron. Lett., vol. 38, pp. 1669-1670, 2002.

[3] D. Taillaert, P. Bienstman, and R. Baets, "Compact efficient broadband grating coupler for silicon-on-insulator waveguides," Opt. Lett., vol. 29, pp. 2749-2751, 2004.

[4] F. Van Laere, M. Ayre, D. Taillaert, D. Van Thourhout, T. F. Krauss, and R. Baets, "Compact and efficient fibre-to-waveguide grating couplers in InP-membrane," Electron. Lett., vol. 42, pp. 343-345, 2006.

[5] B. Wang, J. H. Jiang, and G. P. Nordin, "Compact slanted grating couplers," Opt. Express, vol. 12, pp. 3313-3326, 2004.

[6] _ _ "Embedded, slanted grating for vertical coupling between fibers and silicon-on-insulator planar waveguides," IEEE Photon. Technol. Lett., vol. 17, no. 9, pp. 1884-1886, Sep. 2005.

[7] M. Hagberg, N. Eriksson, and A. Larsson, "Investigation of high-efficiency surface-emitting lasers with blazed grating outcouplers," IEEE J. Quantum Electron., vol. 32, no. 9, pp. 1596-1605, Sep. 1996.

[8] P. Bienstman and R. Baets, "Optical modelling of photonic crystals and VCSELs using eigenmode expansion and perfectly matched layers," Opt. Quantum Electron., vol. 33, pp. 327-341, 2001.

[9] M. V. Kotlyar, L. O'Faolain, R. Wilson, and T. F. Krauss, "High-aspect-ratio chemically assisted ion-beam etching for photonic crystals using a high beam voltage-current ratio," J. Vacuum Sci. Tech. B, vol. 22, pp. 1788-1791, 2004. 